\title{
Mammary Analogue Secretory Carcinoma of the Soft Palate: A Report of Two Cases
}

\author{
Denrick Cooper $^{1}$, Brian Burkey ${ }^{1}$, Deborah Chute ${ }^{2}$, Joseph Scharpf ${ }^{{ }^{*}}$ \\ ${ }^{1}$ Department of Otolaryngology, Head and Neck Surgery, Cleveland Clinic, Cleveland, USA \\ ${ }^{2}$ Department of Anatomic Pathology, Cleveland Clinic, Cleveland, USA \\ Email: *Scharpj@ccf.org
}

Received June 3, 2013; revised June 30, 2013; accepted July 10, 2013

Copyright (C) 2013 Denrick Cooper et al. This is an open access article distributed under the Creative Commons Attribution License, which permits unrestricted use, distribution, and reproduction in any medium, provided the original work is properly cited.

\begin{abstract}
Mammary analogue secretory carcinoma (MASC) is a recently identified salivary tumor that shares morphological features of secretory carcinoma of the breast, salivary acinic cell carcinoma, low grade cystadenocarcinoma and mucoepidermoid carcinoma. To date, a majority of cases have been reported in the major salivary glands with very few seen in the palate. We present 2 single institution cases of MASC of the soft palate to help further characterize this novel tumor.
\end{abstract}

Keywords: Mammary Analogue Secretory Carcinoma; ETV6-NTRK3; Secretory Carcinoma

\section{Introduction}

Salivary gland tumors are relatively rare malignancies with a worldwide annual incidence of 3 per 100,000 people [1]. In the US, salivary gland cancer comprises $6 \%$ of all head and neck cancers and $0.3 \%$ of all malignancies [2]. Mammary analogue secretory carcinoma (MASC) has been a recently described salivary tumor that shares morphological features of secretory carcinoma of the breast, salivary acinic cell carcinoma, low grade cystadenocarcinoma and mucoepidermoid carcinoma [3]. Before its discovery, MASC was often misinterpreted as acinic cell carcinoma due to similarities in their morphologic appearance [4]. In 2010, Skalova et al. distinguished MASC from acinic cell carcinoma based on distinctive molecular alteration. The ETV6-NTRK3 gene fusion, caused by a genetic translocation involving chromosomes 15 and 12, specifically $t(12 ; 15)$ (p13; q25), has become a hallmark factor for differentiating MASC tumors from other salivary gland carcinomas [3,5]. Even with the influx of research on MASC, there is still very little known about the tumor. To date, there have been approximately 70 reported cases of MASC5. A majority of cases have been reported in the major salivary glands (parotid and submandibular), with very few arising from minor glands. Currently, only six cases have been reported in the palate [6]. In this paper, we present 2 single institution cases of MASC of the soft palate to further

*Corresponding author. elucidate this novel cancer.

\section{Case Reports}

\subsection{Case 1}

A 43-year-old Caucasian gentleman presented to the Cleveland Clinic Head and Neck Institute with a several week history of a non-painful, slow growing, left palatal lesion with a bluish hue. The patient could not further specify how long the lesion had been present. He described no difficulty swallowing; however, he did report a feeling of mass effect. The patient had a medical history significant for lung carcinoma with right pneumonectomy (2004) and recurrent papillomatosis of the larynx and trachea, status post three laser surgeries. His physical examination revealed a $2.5 \mathrm{~cm}$ cystic-appearing lesion with a bluish hue on the left soft palate. There was no impingement of the airway. The nose and nasopharynx were free of disease. The neck was without any masses, adenopathy nor thyroid nodules bilaterally.

A computerized tomography scan with contrast, showed a hypodense lesion in the posterior lateral aspect of the left soft palate measuring $2.1 \times 1.7 \mathrm{~cm}$ (Figure 1). No pathological lymphadenopathy was appreciated. confirm that you have the correct template for your paper size.

Fine needle aspiration of the mass revealed cytological findings that were negative for malignant cells but with limited cellularity and consisted primarily of cyst con- 


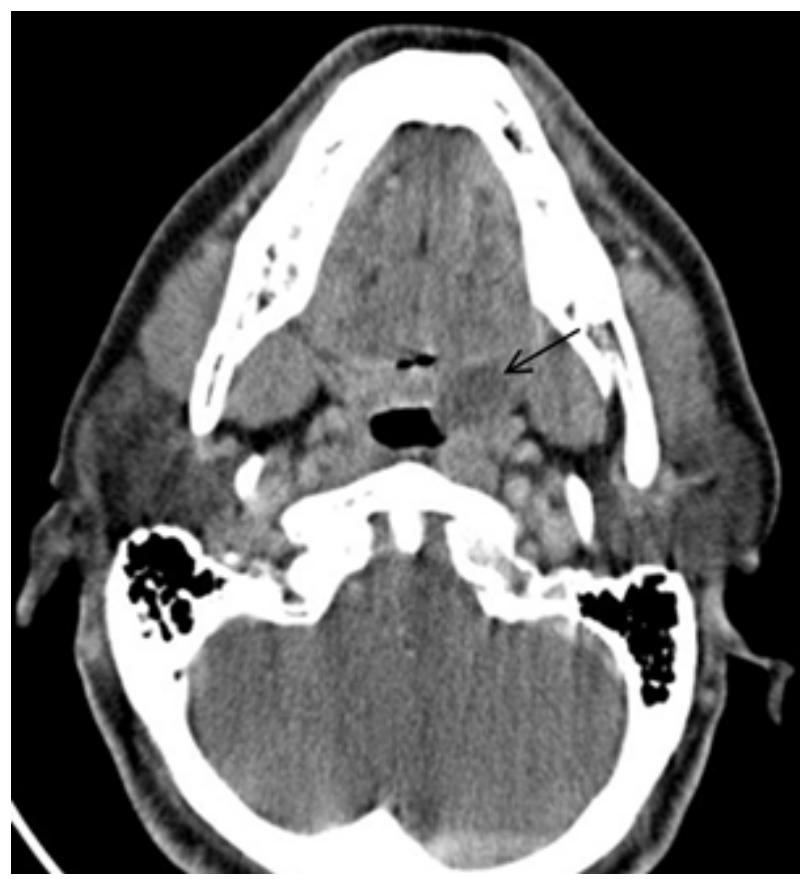

Figure 1. Axial computerized tomography scan with contrast. The black arrow identifies the mass in the left soft palate.

tents. Surgical excision and reconstruction with a right hard palate mucoperiosteal palatal flap was performed in August 2011. Intraoperative evaluation revealed low grade salivary gland malignancy, with definitive classification deferred to permanent section, with negative hard palate and anterior mucosal margins. There has been no disease recurrence in the 14 months since surgery.

\subsection{Case 2}

A previously healthy, 26-year-old Caucasian female presented to Cleveland Clinic with a 7 month history of a raised, violaceous mass on her right soft palate at the junction of the hard palate and soft palate. The asymptomatic mass had slightly increased in size over the prior 6 months before presentation. The patient was previously evaluated by an oral surgeon where an incisional biopsy had been performed. The biopsy was initially interpreted at an outside hospital as acinic cell carcinoma, and extended to the edge of the $1 \times 0.5 \mathrm{~cm}$ specimen. The patient was sent to Cleveland Clinic for further management.

A computerized tomography scan of the head and neck with contrast revealed neither gross tissue abnormalities nor significant lymphadenopathy.

Definitive surgical excision of the mass was performed in April 2011, using a trans-oral robotic surgical approach. The resected specimen measured $1.2 \times 0.5 \times 0.3$ $\mathrm{cm}$. The final pathologic analysis revealed residual tumor measuring $0.7 \mathrm{~cm}$ in size. Intraoperative evaluation showed negative circumferential and deep margins. There has been no disease recurrence in the 24 months since surgery

\section{Results}

\subsection{Case 1}

Pathologic examination of the palate resection demonstrated a well circumscribed, partially cystic, submucosal mass measuring $1.4 \mathrm{~cm}$. Microscopically, the nodule was comprised of microcystic, tubular and papillary structures with a lobulated growth pattern. Abundant eosinophilic luminal material was present. The cells had lowgrade vesicular nuclei with centrally located nucleoli, surrounded by finely vacuolated eosinophilic cytoplasm (Figure 2(a)). Rare mitotic figures were identified. A portion of the tumor had undergone hemorrhagic cystic degeneration. No tumor necrosis, pleomorphism, or atypical mitotic figures were identified.

The tumor was sent to the University of Pittsburgh Medical Center for confirmatory fluorescence in situ hybridization (FISH) studies. According to the University of Pittsburgh's report, the ETV6 FISH analysis (ETV6 dual-color break apart probe, Abbot Molecular, Des Plains IL) showed $53.6 \%$ of tumor cells (69 counted) were positive for the translocation, and $20.3 \%$ of tumor cells had a complex arrangement involving ETV6. The MAML FISH analysis (MEC Dual color break-apart probe, ZytoVision, Bremerhaven, Germany) showed $96.8 \%$ of tumor cells (63 counted) were negative for the translocation, which is considered negative. These results confirmed the diagnosis of MASC.

\subsection{Case 2}

Pathologic examination of the palate resection demonstrated a well circumscribed submucosal nodule measuring $0.7 \mathrm{~cm}$. Microscopically, the nodule was comprised of anastomosing tubular structures with abundant eosinophilic secretory material within the luminal spaces (Figure 2(b)). The cells had low-grade vesicular nuclei with centrally located nucleoli, surrounded by finely granular eosinophilic cytoplasm. Rare mitotic figures were identified, with up to 3 mitotic figures per 10 high power fields. No necrosis, pleomorphism, or atypical mitotic figures were identified.

Immunohistochemical stains demonstrated the neoplastic cells were strongly and diffusely positive for CAM5.2, S-100, and vimentin, and focally positive for CK5/6 and epithelial membrane antigen. The cells were negative for DOG-1, p63, SMA, SMMS-1, and androgen receptor. PAS and PAS with diastase highlighted the luminal secretory material, but were negative within the cell cytoplasm. A mucicarmine stain was negative for intracytoplasmic mucin. 


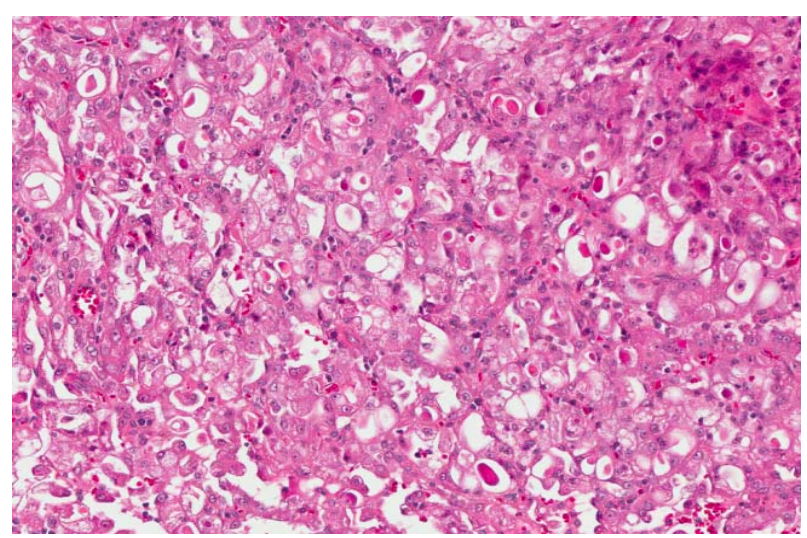

(a)

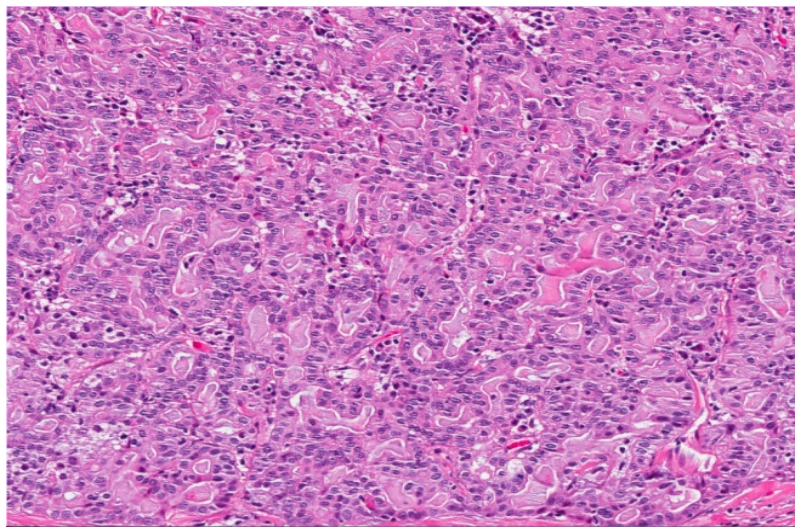

(b)

Figure 2. (a) The tumor from Case 1 contains anastomosing tubular structures composed of low-grade cells with vesicular nuclei and shows abundant eosinophilic secretory material within the luminal spaces (Hematoxylin and Eosin, $400 \times$ ). The tumor from Case 2 (b) shows microcystic and tubular structures composed of cells with low-grade vesicular nuclei surrounded by finely vacuolated eosinophilic cytoplasm, as well as abundant eosinophilic secretory material within the luminal spaces (Hematoxylin and Eosin, 200×).

The tumor was sent to the University of Pittsburgh Medical Center for confirmatory fluorescence in situ hybridization (FISH) studies. According to the University of Pittsburgh's report, the ETV6 FISH analysis (EVT6 dual-color break apart probe, Abbott Molecular, Des Plains IL), showed $81.7 \%$ of tumor cells (60 counted) were positive for the translocation. These results confirmed the diagnosis of MASC.

\section{Discussion}

MASC represents a relatively new tumor classification for malignancies previously commonly classified as acinic cell cancers [4]. Histologically, MASC bears resemblance to secretory breast cancer and some salivary gland tumors, particularly acinic cell carcinoma and mucoepidermoid carcinoma. This should not be surprising since salivary and breast tissues derive from the same ecto- dermal embryonic origin and perform similar functions in the body.

Since the designation of MASC, there have been over 70 clinical cases in the literature. A majority of the cases present in the major salivary glands, primarily the parotid. Very few cases have been seen in the soft palate (6 in all) $[5,7,8]$. This report adds an additional two cases of MASC of the soft palate to the literature. Both patients presented with tumors which appeared cystic and lobulated on physical examination. Microscopically, the tumor cells were arranged in microcystic, glandular and papillary patterns with abundant vacuolated eosinophilic cytoplasm. This morphology is consistent with MASC as previously described in the literature [5,8-11]. In addition, FISH studies demonstrated the ETV6-NTRK3 gene fusion diagnostic for MASC in both cases.

Due to its novel nature, the clinical and pathological features of MASC are still being fully elucidated. However, retrospective studies have found clinical presentations similar to the two presented in our study. MASC can occur over a very wide age range, from 14 - 77 years old, and often presents as a painless mass for a varied amount of time, ranging from 2 months to several years. MASC is more common in men, with a male:female ratio of 1.5:1. Currently, the literature has not shown a predilection towards race. Patients from Cases 1 and 2 presented with nodules described as painless and slowly growing for several weeks and 7 months respectively [5$7,11,12]$. The violaceous and blue hues of the masses, seen on physical exam, have not been recorded in prior MASC of the soft palate reports.

Risk factors for this malignancy remain unknown. Both patients in this report had no prior history of smoking, alcohol intake or other risk factors associated with oral cancers. The patient from Case 1, did however, have a history of respiratory papillomatosis of the larynx, caused by human papilloma virus.

In addition to AciCC, the differential diagnosis for MASC includes polymorphous low-grade adenocarcinoma (PLGA) and low-grade cribriform cystadenocarcinoma (LGCCC). Similar to MASC, PLGA can arise from the minor salivary glands and has an indolent growth pattern with rare invasion into local structures [13]. Grossly, PLGA is well circumscribed, unencapsulated and usually not seen with hemorrhage or necrosis [14]. Its microscopic presentation makes it especially difficult to differentiate because of the mixture of tubular, papillary, cribriform, cystic and solid growth patterns that can be seen at presentation. Histologically, both MASC and PLGA stain for S-100 and cytokeratin In contrast to PLGA, MASC is largely eosinophilic and weakly stains for Ki-67, while PLGA strongly stains for Ki-67 and GFAP $[9,15,16]$. The genetics of PLGA are still poorly understood causing genetic markers to be useless in its 
diagnosis. The ETV-NTRK3 fusion transcript is pathognomonic for MASC.

Low-grade cribiform cystadenocarcinoma (LGCCC) also has a similar clinical course, the architecture and staining pattern compared to MASC. Both share indolent courses and can vary in architecture presenting with cystic, solid, cribiform and papillar structures. LGCCC and MASC both stain positive for S100 and CK7. In contrast, the myoepithelial markers, SMA and calponin are specific to LGCCC $[17,18]$. As previously mentioned, the ETV6-NTRK3 translocation is specific to MASC

Since most MASC cases were previously diagnosed as acinic cell carcinomas, many were treated with the same surgical intervention as if acinic cell carcinoma. Similar to previous cases of MASC, the current two patients were treated with surgical excision and reconstruction with no recurrence of the disease following at least 1.5 years follow-up. In a retrospective study done by Chiosea et al. $(n=27)$, the disease free survival of conventional MASC versus acinic cell carcinoma showed no significant statistical difference [7]. Rare recurrences (8 in all), and one case of metastasis have been reported [5]. However, it is not yet known whether these cases were caused by a more aggressive form of MASC.

With the small number of cases published, it is difficult to draw conclusions regarding the treatment of MASC. Surgical intervention has been the primary treatment thus far. Research has focused on a molecular level and on the ETV6-NRTK3 translocation. Tognon et al., found that the IGF1R axis is vital for the oncogenic transformation of fibroblasts containing the translocation. Moreover, IGIFR/INSR kinase inhibitors have been proven to be effective in significantly reducing tumor growth in vivo and in vitro. These treatments potentially could allow for a targeted therapy management strategy for this cancer.

In summary, this report adds two additional cases of MASC, involving the soft palate, that the body of literature is currently available for this newly described entity. The clinical presentations and morphologies of these cases are similar to previously reported MASC cases. However, the bluish and violaceous hues, seen in Cases 1 and 2 respectively have not been reported in previous MASC cases of the soft palate. These observations will help further characterize this novel tumor.

\section{REFERENCES}

[1] D. M. Parkin, J. Ferlay, M. P. Curado, et al., "Fifty Years of Cancer Incidence: CI5 I-IX,” International Journal of Cancer, Vol. 127, No. 12, 2010, pp. 2918-2927. doi:10.1002/ijc.25517

[2] L. A. G. Ries, B. F. Hankey, B. A. Miller, et al., "Cancer Statistics Review 1973-88,” National Cancer Institute, Bethesda, 1991.
[3] C. Tognon, S. R. Knezevich, D. Huntsman, et al., "Expression of the ETV6-NTRK3 Gene Fusion as a Primary Event in Human Secretory Breast Carcinoma," Cancer Cell, Vol. 2, No. 5, 2002, pp. 367-376. doi:10.1016/S1535-6108(02)00180-0

[4] S. I. Chiosea, et al., "The Profile of Acinic Cell Carcinoma after Recognition of Mammary Analog Secretory Carcinoma," American Journal of Surgical Pathology, Vol. 36, No. 3, 2012, pp. 343-350.

doi:10.1097/PAS.0b013e318242a5b0

[5] A. Skalova, T. Vanecek, R. Sima, et al., "Mammary Analogue Secretory Carcinoma of Salivary Glands, Containing the ETV6-NTRK3 Fusion Gene: A Hitherto Undescribed Salivary Gland Tumor Entity,” American Journal of Surgical Pathology, Vol. 34, No. 5, 2010, pp. 599-608. doi:10.1097/PAS.0b013e3181d9efcc

[6] F. J. Kratochvil 3rd, J. C. Stewart and S. R. Moore, "Mammary Analog Secretory Carcinoma of Salivary Glands: A Report of 2 Cases in the Lips,” Oral Surgery, Oral Medicine, Oral Pathology and Oral Radiology, Vol. 114, No. 5, 2012, pp. 630-635. doi:10.1016/j.0000.2012.07.480

[7] S. I. Chiosea, C. Griffith, A. Assaad and R. R. Seethala, "Clinicopathological Characterization of Mammary Analogue Secretory Carcinoma of Salivary Glands,” Histopathology, Vol. 61, No. 3, 2012, pp. 387-394. doi:10.1111/j.1365-2559.2012.04232.x

[8] A. Fehr, T. Loning and G. Stenman, "Mammary Analogue Secretory Carcinoma of the Salivary Glands with ETV6-NTRK3 Gene Fusion,” American Journal of Surgical Pathology, Vol. 35, No. 10, 2011, pp. 1600-1602. doi:10.1097/PAS.0b013e31822832c7

[9] F. Petersson, D. Lian, Y. P. Chau and B. Yan, "Mammary Analogue Secretory Carcinoma: The First Submandibular Case Reported Including Findings on Fine Needle Aspiration Cytology,” Head and Neck Pathology, Vol. 6, No. 1, 2012, pp. 135-139. doi:10.1007/s12105-011-0283-x

[10] L. Pisharodi, "Mammary Analog Secretory Carcinoma of Salivary Gland: Cytologic Diagnosis and Differential Diagnosis of an Unreported Entity,” Diagnostic Cytopathology, Vol. 41, No. 3, 2012, pp. 239-243. doi: $10.1002 /$ dc. 21766

[11] S. Ito, E. Ishida, A. Skalova, K. Matsuura, H. Kumamoto and I. Sato, "Case Report of Mammary Analog Secretory Carcinoma of the Parotid Gland," Pathology International, Vol. 62, No. 2, 2012, pp. 149-152. doi:10.1111/j.1440-1827.2011.02759.x

[12] J. C. Rastatter, K. R. Jatana, L. J. Jennings and H. MelinAldana, "Mammary Analogue Secretory Carcinoma of the Parotid Gland in a Pediatric Patient, Otolaryngology -Head and Neck Surgery, Vol. 146, No. 3, 2012, pp. 514-515. doi:10.1177/0194599811419044

[13] S. D. Vincent, H. L. Hammond and M. W. Finkelstein, "Clinical and Therapeutic Features of Polymorphous LowGrade Adenocarcinoma,” Oral Surgery, Oral Medicine, Oral Pathology, Vol. 77, No. 1, 1994, pp. 41-47. doi:10.1016/S0030-4220(06)80105-2

[14] R. González-García, F. J. Rodríguez-Campo, M. F. Muñoz-Guerra, S. H. Nam-Cha, J. Sastre-Pérez and L. Na- 
val-Gías, "Polymorphous Low-Grade Adenocarcinoma of the Palate: Report of Cases," Auris, Nasus, Larynx, Vol. 32, No. 3, 2005, p. 275. doi:10.1016/j.anl.2005.03.019

[15] J. T. Castle, L. D. Thompson, R. A. Frommelt, B. M. Wenig and H. P. Kessler, "Polymorphous Low Grade Adenocarcinoma," Cancer, Vol. 86, No. 2, 1999, pp. 207-219.

doi:10.1002/(SICI)1097-0142(19990715)86:2<207::AIDCNCR4>3.0.CO;2-Q

[16] I. A. El-Naaj, Y. Leiser, A. Wolff and M. Peled, "Polymorphous Low Grade Adenocarcinoma: Case Series and Review of Surgical Management," Journal of Oral and
Maxillofacial Surgery, Vol. 69, No. 7, 2011, pp. 19671972. doi:10.1016/j.joms.2010.10.010

[17] M. Brandwein-Gensler and D. R. Gnepp, "Low-Grade Cribriform Cystadenocarcinoma,” World Health Organization Classification of Tumours, Pathology \& Genetics, Head and Neck Tumours, 2005.

[18] L. Wang, Y. Liu, X. Lin, D. Zhang, Q. Li, X. Qiu and E. H. Wang, "Low-Grade Cribriform Cystadenocarcinoma of Salivary Glands: Report of Two Cases and Review of the Literature," Diagnostic Pathology, Vol. 8, No. 1, 2013, pp. 1-6. doi:10.1186/1746-1596-8-28 